through state and territory arthritis foundations. In addition to receiving the ASMC, patients received routine care for their arthritis. Efficacy data were collected before and 6 months and 2 years after completing the ASMC.

The study showed that the ASMC led to a sustained improvement in health status 2 years after the course was completed. Patients had a small reduction in levels of pain and fatigue, a reduction in health distress, and an increase in self-efficacy. No progression in disability was observed over the 2-year period, and the number of patients who were performing aerobic walking was higher at 2 years than at baseline. No adverse events were reported. These findings were consistently observed across demographic groups. The authors conclude that although the average program benefits were small, at the population level these benefits could be substantial.

Original article Osborne RH et al. (2007) Does selfmanagement lead to sustainable health benefits in people with arthritis? A 2-year transition study of 452 Australians. J Rheumatol 34: 1112-1117

\section{Smoking increases the risk of cartilage loss in individuals with a family history of OA}

Whether smoking contributes to the pathogenesis of osteoarthritis (OA) is unclear, because both protective and harmful effects have been reported. Limitations in defining any role of smoking might result from variability in the way radiographic measurements are made to assess OA, and the inability of radiography to detect cartilage defects that can precede cartilage loss and ultimately lead to OA. For these reasons Ding et al. used MRI, which can detect cartilage defects and volume changes, to assess the effect of smoking on knee cartilage. The authors also tested for any interaction between smoking and family history of OA for the development of these cartilage changes.

In this longitudinal study, MRI scans of the right knee were obtained (at baseline and on average 2.3 years later) from adult offspring of at least one parent with severe knee OA $(n=162)$ and randomly selected control individuals $(n=163)$ with no parental history of knee $\mathrm{OA}$; the mean age of participants was 45 years. Questionnaire information on smoking history was obtained only at baseline when other measures of OA such as knee pain were also assessed. Smoking did not appear to be associated with cartilage defects or loss of volume in the control individuals; however, smoking increased the risk of knee cartilage defects and loss of volume before and after adjustment for established confounders in individuals who had a family history. The small size of the smoking subgroups was a study limitation.

Smoking negatively affects knee cartilage health in offspring of patients with severe knee OA.

Original article Ding C et al. (2007) Smoking interacts with family history with regard to change in knee cartilage volume and cartilage defect development. Arthritis Rheum 56: $1521-1528$

\section{Multiplex immunoassay could be valuable for the diagnosis of connective tissue diseases}

Antinuclear antibody (ANA) tests are widely used in the initial evaluation of patients suspected of having connective tissue disease (CTD); however, this approach has a high false-positive rate. To overcome this limitation, the multiplex immunoassay has been developed to evaluate multiple CTD-specific autoantibodies from a single specimen. This assay employs a Medical Decision Support Software, which compares test results to antibody patterns associated with specific CTDs, to predict a diagnosis. Moder et al. have now compared the accuracy of the multiplex immunoassay with that of established enzyme immunoassays (EIAs) for diagnosing and classifying CTD.

Serum samples were collected from 908 patients ( $83 \%$ female) with highly suspected or previously diagnosed CTD at 3 US rheumatology clinics. Each sample was analyzed with a multiplex immunoassay and EIAs for the presence of ANA, as well as for antibodies specific to SLE, scleroderma, Sjögren's disease and other CTDs. A limitation of the multiplex immunoassay was the absence of antibodies specific to RA.

Of the patients included in the study, $30 \%$ had RA, $37 \%$ had SLE, and $5 \%$ had scleroderma. Overall, the multiplex immunoassay gave comparable results to the individual EIAs for most of the disease-specific autoantibodies. The multiplex immunoassay did, however, exhibit 